Supporting Information

\title{
Imprinting Tunable $\pi$-magnetism in Graphene Nanoribbons via Edge Extensions
}

\author{
Michele Pizzochero* and Efthimios Kaxiras \\ School of Engineering and Applied Sciences, Harvard University, \\ Cambridge, Massachusetts 02138, USA \\ E-mail: mpizzochero@g.harvard.edu
}




\section{Supplementary Notes}

\section{Supplementary Note 1: Computational Details}

The Kohn-Sham orbitals of the valence electrons are represented as a linear combination of atom-centered basis functions of double- $\zeta$ plus single polarization (DZP) quality, while core electrons are replaced by norm-conserving pseudopotentials. A mesh-cutoff of $400 \mathrm{Ry}$ is used throughout. For the integration over the one-dimensional Brillouin zone, we employ the equivalent of $36 k$-points per single 7-AGNR unit cell in all our calculations but those of the electronic density of states and transport properties, for which a denser grid of 500 and $300 \mathrm{k}$-points is adopted, respectively. The atomic coordinates are optimized until the maximum component of the atomic forces is smaller than $0.02 \mathrm{eV} / \AA$. We introduce single or pairs of edge extensions [Fig. 1(a-d)] in otherwise bare supercells of 7-AGNR containing 324 atoms and of length equals to $7.76 \mathrm{~nm}$. The periodic arrangement of the edge extensions, giving rise to the sawtooth graphene nanoribbon shown in Fig. 1(e), is modeled with a unit cell $2.59 \mathrm{~nm}$ long, the Brillouin zone of which is sampled with the equivalent of $18 k$-points per AGNR unit cell. In all models, carbon atoms at the edges of graphene nanoribbons are saturated with hydrogen atoms, in analogy with experiments. Vacuum regions of $\sim 1.0 \mathrm{~nm}$ or larger are introduced to avoid artificial interactions between periodic replicas.

We obtain the conductance spectra by means of the non-equilibrium Green's functions

$$
\tilde{G}(E)=\left[E I-H-\Sigma_{L}(E)-\Sigma_{R}(E)\right]^{-1},
$$

where $\tilde{G}(E)$ is the Green's function, $E$ is the energy, $I$ is the identity matrix, and $\Sigma_{L(R)}$ is the self-energy of the semi-infinite left (right) lead. Such self-energies are determined self-consistently as

$$
\Sigma_{L(R)}(E)=H_{1}^{\dagger}\left[E I-H_{0}-\Sigma_{L(R)}(E)\right]^{-1} H_{1}
$$

with $H_{0}$ being the Hamiltonian of the unit cell in the lead, $H_{1}$ their coupling, and $\Gamma_{L(R)}$ the 
broadening function

$$
\Gamma_{L(R)}(E)=i\left[\Sigma_{L(R)}(E)-\Sigma_{L(R)}(E)^{\dagger}\right]
$$

Finally, the conductance $G(E)$ - in units of conductance quantum $G_{0}$ - is achieved through the Landauer formula

$$
G(E)=G_{0} T(E)=\frac{2 e^{2}}{h} T(E)=\frac{2 e^{2}}{h} \operatorname{Tr}\left[\Gamma_{L} \tilde{G} \Gamma_{R} \tilde{G}^{\dagger}\right]
$$

where $e$ and $h$ are the elementary charge and the Planck constant, respectively. In the conductance spectra given in Figs. 2(c) and 3(c), we adopted 7-AGNRs as right and left semi-infinite leads.

\section{Supplementary Note 2: Stability of the edge-extensions in 7-AGNR}

Although the surface-assisted synthesis of the edge extensions in 7-AGNR studied in this work mainly proceeds under kinetic control, it is nevertheless instructive to analyze the relative stability of the resulting reaction products. To this end, we obtain the formation energy $E_{\text {form }}(\mu)$, in which the dependence on the chemical potential $\mu$ is dictated by the binary nature (carbon and hydrogen) of graphene nanoribbons. As is customary, we rely on the following expression

$$
E_{\mathrm{form}}(\mu)=\frac{1}{\alpha}\left[E_{\mathrm{GNR}+\mathrm{E}}+E_{\mathrm{GNR}}+n_{\mathrm{C}} \mu_{\mathrm{C}}+n_{\mathrm{H}} \mu_{\mathrm{H}}\right]
$$

where $E_{\mathrm{GNR}+\mathrm{E}}$ and $E_{\mathrm{GNR}}$ are the total energies of 7-AGNR with and without a number $\alpha$ of naphtho groups, respectively, $n_{\mathrm{C}}$ and $n_{\mathrm{H}}$ the amount of atoms of $\mathrm{C}$ and $\mathrm{H}$ atoms that have to be added to 7 -AGNR to create the edge extension, and $\mu_{\mathrm{C}}$ and $\mu_{\mathrm{H}}$ their corresponding chemical potentials. We assume that $\mathrm{C}$ and $\mathrm{H}$ are in thermal equilibrium with a 7 -AGNR unit cell of chemical formula $\mathrm{C}_{14} \mathrm{H}_{4}$ through the equality $\mu_{\mathrm{C}_{14} \mathrm{H}_{4}}=14 \mu_{\mathrm{C}}+4 \mu_{\mathrm{H}}$, in which graphene

and molecular hydrogen $\left(\mathrm{H}_{2}\right)$ are assumed to be the reference systems for the determination 
of the chemical potential reservoirs. The resulting formation energies are equal to $1.35 \mathrm{eV}$ $(1.32 \mathrm{eV}), 1.34 \mathrm{eV}(1.31 \mathrm{eV})$, and $1.20 \mathrm{eV}(1.17 \mathrm{eV})$ in C-rich (H-rich) condition for the $D_{1}, D_{2}, D_{3}$ dimer configuration [see Fig. 1(b-d)], respectively, thereby suggesting that the $D_{2}$ dimer is the thermodynamically stable one. The comparison of these formation energies with those of an individual fused naphtho group [see Fig. 1(a)], i.e., $1.38 \mathrm{eV}(1.35 \mathrm{eV})$ in C-rich (H-rich) condition, allows us to infer that the aggregation of the edge extensions is unlikely to occur when thermodynamic equilibrium prevails. 


\section{Supplementary Figures}
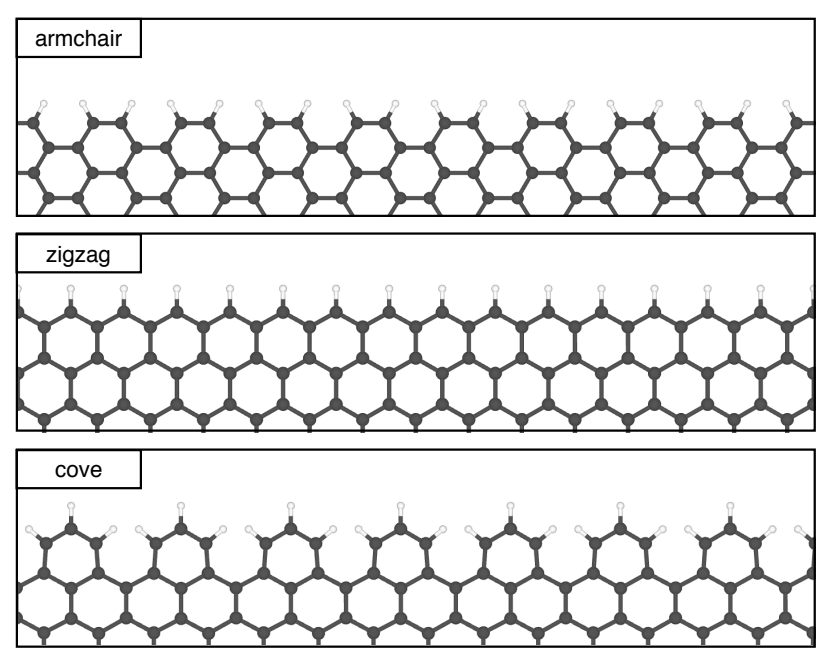

Figure S1: Atomic structure of the three edge geometries considered in this work.
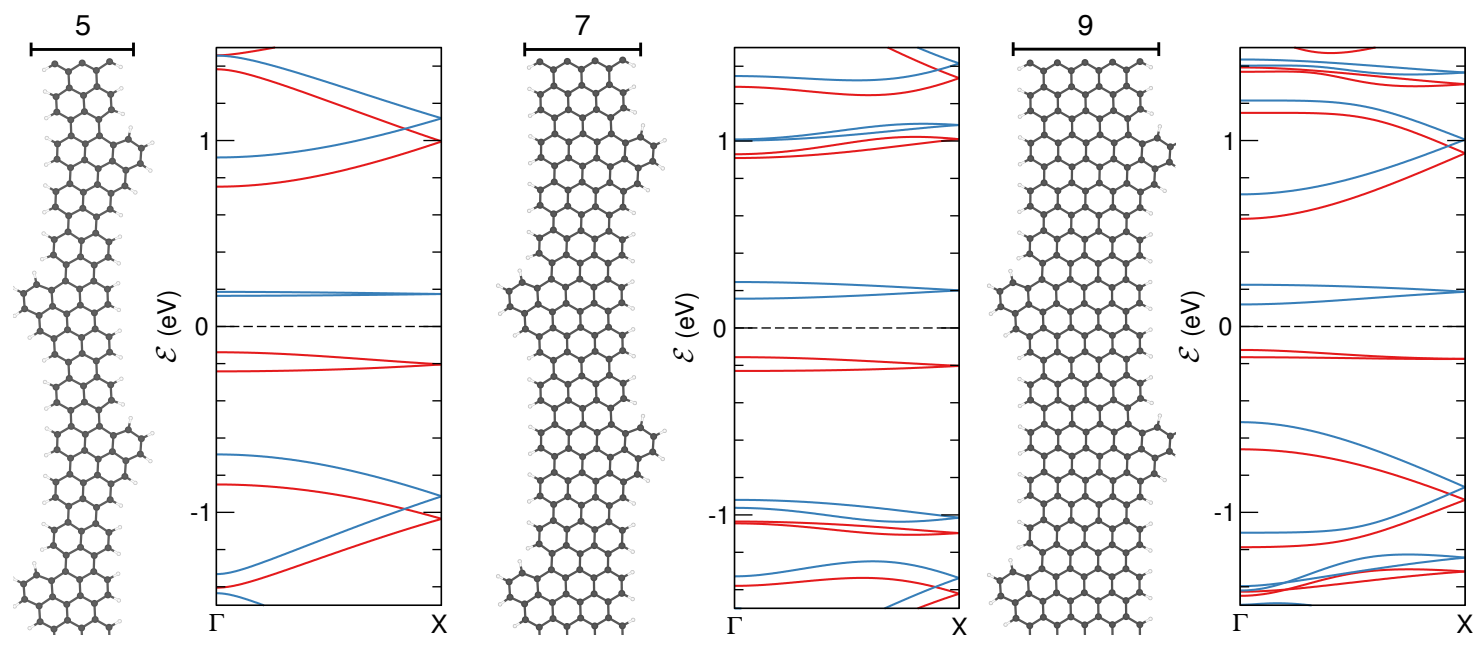

Figure S2: Atomic and band structures of sawtooth graphene nanoribbons in which the array of edge extensions is incorporated in 5-AGNR (left), 7-AGNR (center), and 9-AGNR (right). Red (blue) lines indicate the spin-majority (spin-minority) channels. Dashed horizontal line indicates the Fermi level, to which energies are referenced. These three nanoribbons feature a $S=1$ magnetic ground state and full spin-polarization at the band extrema. 

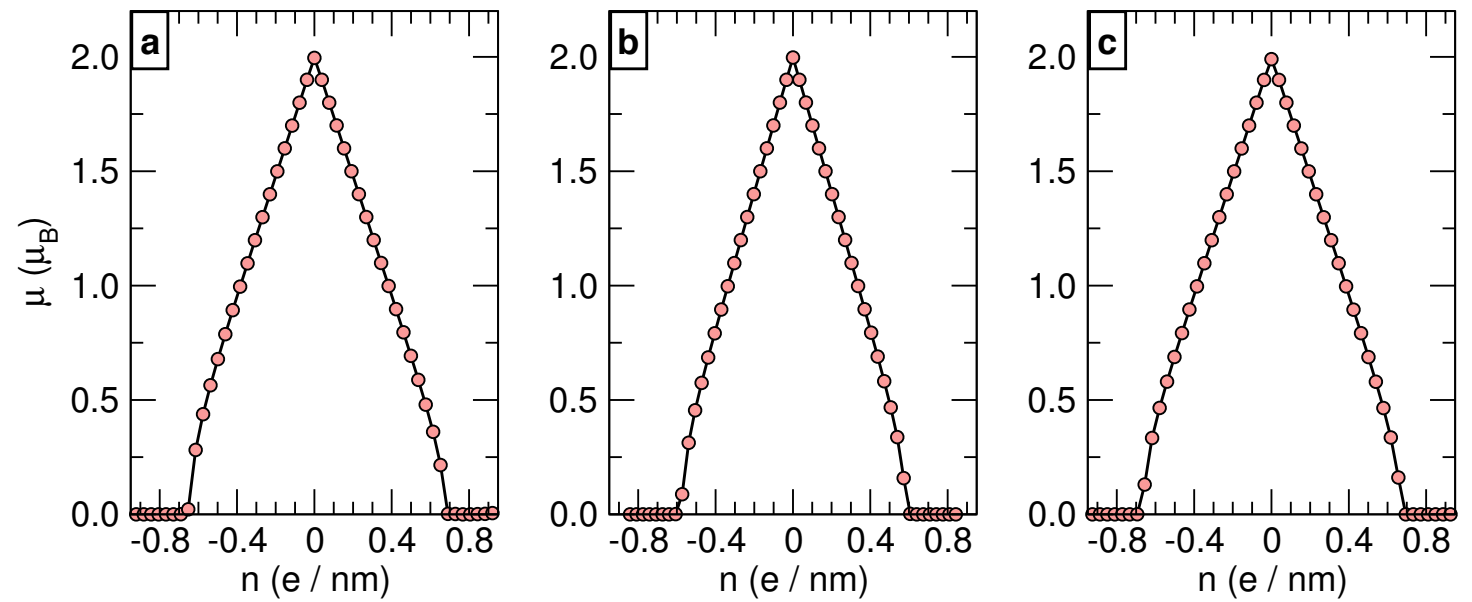

Figure S3: Evolution of the total magnetic moment with charge doping in sawtooth graphene nanoribbons, in which the edge extensions are incorporated in 5-AGNR (left), 7-AGNR (center), and 9-AGNR (right). The magnetism is quenched at $\sim \pm 0.7 \mathrm{e} / \mathrm{nm}$, irrespectively of the width.
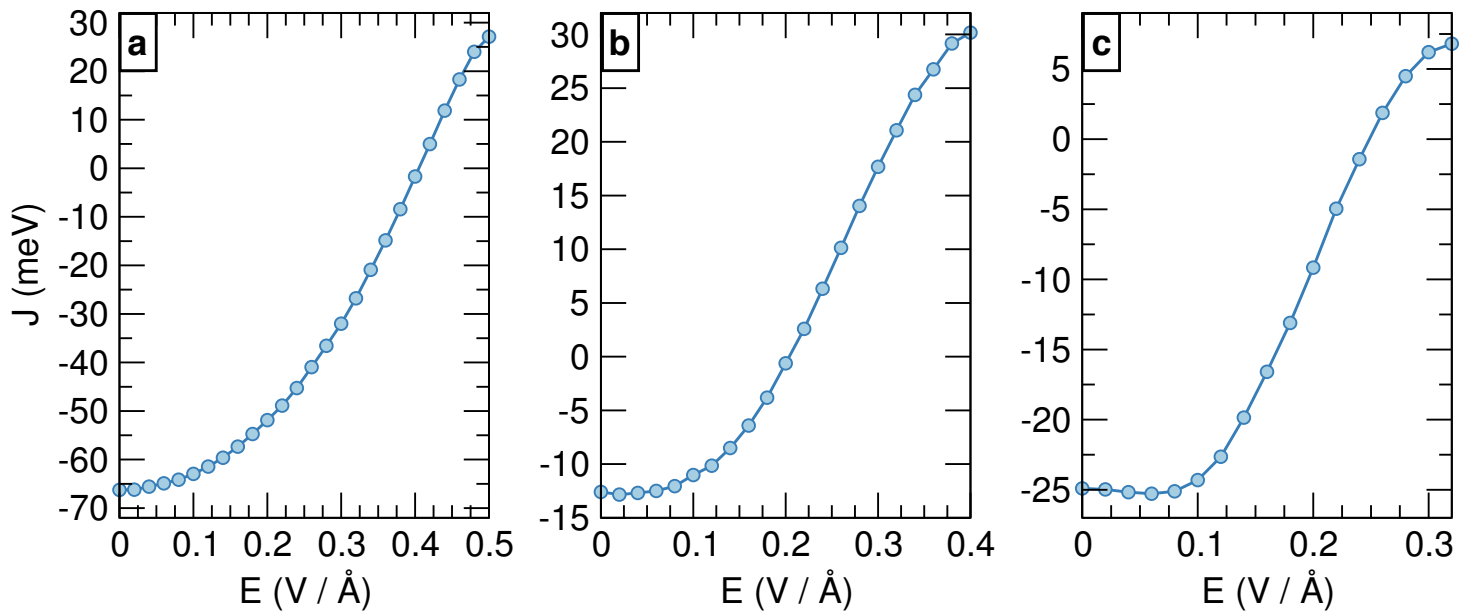

Figure S4: Evolution of the Heisenberg exchange coupling with transverse electric field in sawtooth graphene nanoribbons, in which the edge extensions are incorporated in 5-AGNR (left), 7-AGNR (center), and 9-AGNR (right). A ferromagnetic $(J<0)$ to antiferromagnetic $(J>0)$ phase transition is observed irrespectively of the width. 

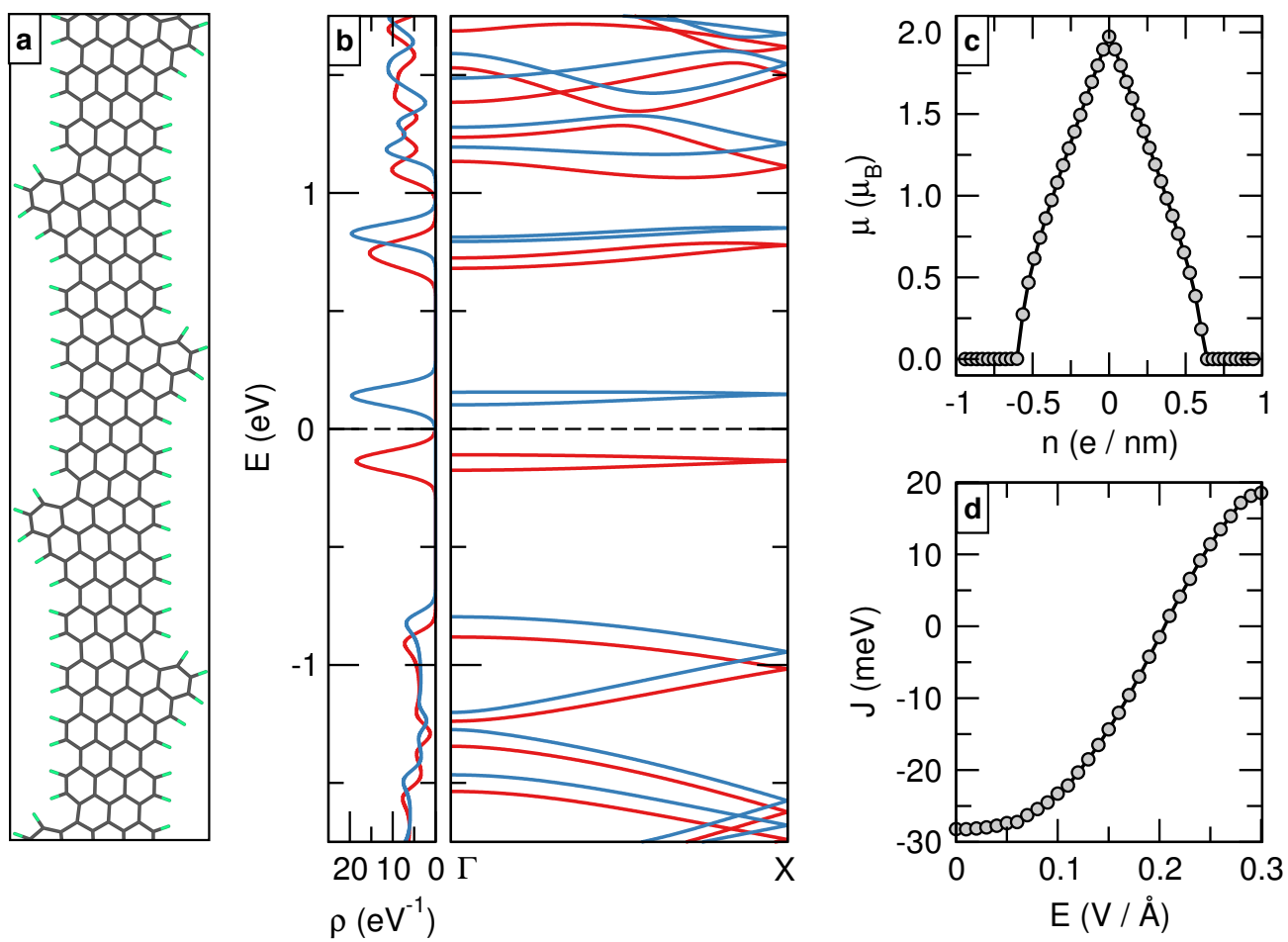

Figure S5: (a) Atomic structure of fluorine-terminated 7-atom wide "sawtooth" graphene nanoribbons (F-sGNR). Carbon (fluorine) atoms are indicated in black (green). This system features a spin-1 ground state, with the edge extensions being ferromagnetically coupled by $J=-28 \mathrm{meV}$. This ferromagnetic solution lies $123 \mathrm{meV}$ lower in energy than the nonmagnetic one. (b) Electronic density of states and band structure of F-terminated sGNR. Red (blue) lines indicate the spin-majority (spin-minority) channel. (c) Evolution of the total magnetic moment in F-terminated sGNR with charge doping. The magnetism is quenched at $\sim-0.55-e / \mathrm{nm}$. (d) Evolution of the Heisenberg exchange coupling in F-terminated sGNR with the transverse electric field. A ferromagnetic $(J<0)$ to antiferromagnetic $(J>0)$ phase transition is observed at $E=0.20 \mathrm{~V} / \AA$. 


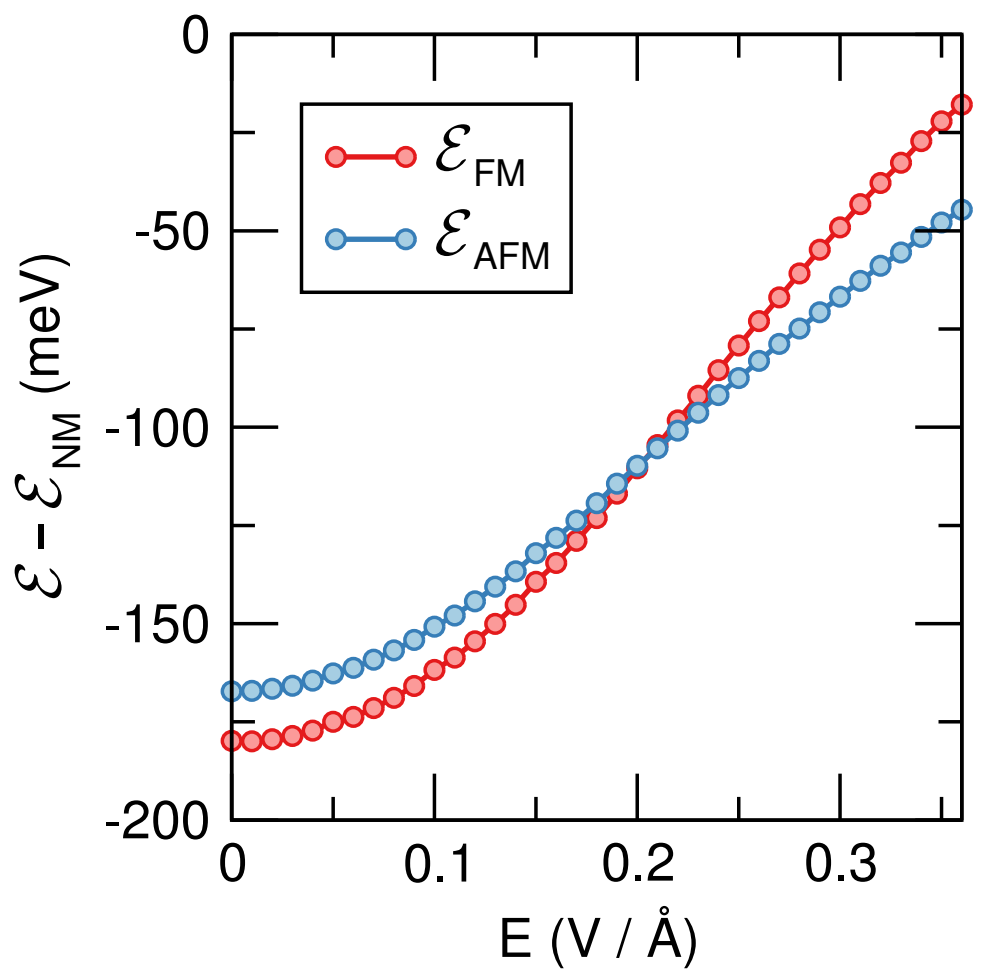

Figure S6: Evolution of the energy of the ferromagnetic and antiferromagnetic state in 7sGNR with a transverse electric field. Values are referenced to the energy of the non-magnetic state at each field. The energy of the non-magnetic state remains higher in energy than that of the magnetic states. 